\title{
Adaptation and selective constraint throughout the Drosophila melanogaster life-cycle
}

\author{
M. Coronado-Zamora ${ }^{1}$, I. Salvador-Martínez ${ }^{2,3}$, D. Castellano ${ }^{4}$, I. Salazar-Ciudad ${ }^{1}$, \\ A. Barbadilla ${ }^{1 *}$ \\ ${ }^{1}$ Genomics, Bioinformatics and Evolution. Departament de Genètica i de Microbiologia, \\ Universitat Autònoma de Barcelona, Cerdanyola del Vallès, Spain \\ ${ }^{2}$ EvoDevo Helsinki Community, Centre of Excellence in Experimental and Computational Developmental \\ Biology, Institute of Biotechnology, University of Helsinki, Helsinki, Finland \\ ${ }^{3}$ Department of Genetics, Evolution and Environment, University College London, London, UK \\ ${ }^{4}$ Bioinformatics Research Center, Aarhus University, Aarhus C, Denmark \\ *e-mail: antonio.barbadilla@uab.es
}

Key words: adaptation, selective constraint, population genomics, transcriptomics, evo-devo

\begin{abstract}
Motivation and Aim: Numerous studies have tried to test whether embryonic development for morphological, gene expression and genomic metrics follows the von Baer [1] or the hourglass [2] hypotheses of embryonic developmental evolution. Here we integrate population genomics data with the developmental transcriptome to measure both adaptation and selective constraint over the embryonic development and life cycle of the species Drosophila melanogaster.
\end{abstract}

Methods: We gather gene expression data for each temporal stage from the modENCODE database. We use three different methods combining genome polymorphism data in D. melanogaster and divergence data between D. melanogaster and D. yakuba. In addition, for each developmental stage, we estimate gene features such as codon usage bias, intron length, expression bias, number of exons and number of transcripts.

Results: (i) Genes expressed in the pupa and adult male stages exhibit the highest levels of adaptive substitutions. (ii) Genes expressed in mid and late embryonic development stages show the highest sequence conservation and the most complex structure: they are larger, with more exons, more transcripts and longer introns. This gene structure complexity may account for the observed conservation. (iii) Earliest stages of embryonic development are the most divergent, but in contrast with the adaptive explanation of pupa and adult stages, this increased divergence seems to be due to the diminished efficiency of natural selection on maternal genes and the simpler gene structure in these stages.

Conclusion: Considered over the whole life-cycle, D. melanogaster seems to fit the hour-glass model of embryonic development evolution.

Acknowledgements: Supported by the Spanish Ministerio de Economía, Industria y Competitividad [Project MINECO CGL2017-89160-P] to AB, the Finnish Academy to IS-C and the Generalitat de Catalunya grant 2017SGR 1379.

\section{References}

1. von Baer K.E. (1828). Über Entwicklungsgeschichte der Thiere. Beobachtung und Reflexion. Königsberg, Bei den Gebrüdern Bornträger.

2. Medawar P.B. (1954). The significance of inductive relationships in the development of vertebrates. J. Embryol. Exp. Morphol. 2:172-174. 